\title{
A NOTE ON ESSENTIAL FINITE INDECOMPOSABILITY AND THICKNESS IN PRIMARY ABELIAN GROUPS
}

\author{
PATRICK W. KEEF AND PETER V. DANCHEV
}

\begin{abstract}
We present a new characterization of essentially finitely indecomposable abelian $p$-groups. Parallel ideas are also applied to the socles of groups, especially in the case of groups that are pure-complete. These results are then used to discuss the class of thick abelian $p$-groups.
\end{abstract}

\section{Introduction}

By the term "group" we will mean an abelian $p$-group, where $p$ is a fixed prime. Our terminology and notation will follow [9]. A group will be said to be $\Sigma$-cyclic if it is isomorphic to a direct sum of cyclic groups.

A group $G$ is said to be essentially finitely indecomposable, or efi for short, if for any decomposition $G=\oplus_{i \in I} G_{i}$, there exists a positive number $m$ such that almost all summands $G_{i}$ are bounded by $p^{m}$. Equivalently, a reduced group $G$ is efi if and only if it does not admit an unbounded $\Sigma$-cyclic direct summand; that is, if $G=A \oplus C$ and $C$ is $\Sigma$-cyclic, then $C$ is bounded.

The main purpose of this note is to give a new criterion for a group to be essentially finitely indecomposable (Theorem 2.1). We then use the terminology of valuated vector spaces to obtain parallel results for the socles of groups (e.g., Proposition 3.2; see [10] for more details about this terminology). If $G$ is a group that is pure-complete (i.e., for every subgroup $S$ of $G[p]$, there is a pure subgroup $P \subseteq G$ such that $P[p]=S$ ), then these two approaches produce comparable results (e.g., Proposition 3.7).

A group $A$ is said to be thick if whenever $H$ is $\Sigma$-cyclic and $f: A \rightarrow H$ is a homomorphism, then $f$ is small (i.e., the kernel of $f$ contains a large subgroup of $A$ ). In view of [11] this is tantamount to the condition that for each homomorphism $f: A \rightarrow H$ with $H$ a $\Sigma$-cyclic group, there exists a positive integer $i$ such that $f\left(\left(p^{i} G\right)[p]\right)=\{0\}$. In our exposition we will use an equivalent formulation from [1]: $A$ is thick if and only if whenever $K$ is a subgroup of $A$ and

Corresponding author: Peter V. Danchev.

2010 Mathematics Subject Classification. 20K10.

Key words and phrases. Primary abelian groups, thick groups, essentially finitely indecomposable groups. 
$A / K$ is $\Sigma$-cyclic, then there is a natural number $j$ such that $\left(p^{j} A\right)[p] \subseteq K$. A useful fact is that $A$ is thick if and only if $A / p^{\omega} A$ is thick (see, e.g., [4]).

Clearly each thick group is efi (otherwise, consider the projection onto an unbounded $\Sigma$-cyclic summand), while the converse is not true (see, e.g., [6] and [5]). Nevertheless, these two classes share some interesting properties; for instance in [2] it was proved that $G$ is efi if and only if $G / p^{\omega} G$ has this property.

We use the our results to give new perspectives on some old results; for example, that a group with socle that is efi is necessarily thick (Corollary 3.6; cf. [12]), and that the converse holds for pure-complete groups (Corollary 3.8; cf. [1]).

\section{A criterion for reduced efi groups}

Recall that a group $G$ is essentially finitely indecomposable, or efi, if $G=\oplus_{i \in I} G_{i}$ implies that there exists a positive integer $m$ such that $p^{m} G_{i}=\{0\}$ for almost all (i.e., for all but a finite number of) indices $i \in I$. For reduced groups, the following gives another characterization.

Theorem 2.1. If $G$ is a reduced group, then $G$ is efi iff whenever $G=\cup_{n<\omega} A_{n}$, where $A_{n} \subseteq$ $A_{n+1} \leq G$ and each $A_{n}$ is pure and nice in $G$, there exists an index $k$ such that $G / A_{k}$ is bounded.

Proof. If $G$ is not efi, then it has an unbounded summand which is $\Sigma$-cyclic. Using this, we can easily express $G$ as an ascending union of a sequence of summands $A_{n}$ of $G$ such that each $G / A_{n}$ is unbounded.

Conversely, suppose the group $G$ can be expressed as the ascending union of a sequence of pure, nice subgroups $A_{n}$ such that each $G / A_{n}$ is unbounded. We want to show that $G$ is not efi.

We construct a strictly increasing sequence of positive integers, $\left\{n_{i}\right\}_{i<\omega}$, elements $x_{i} \in A_{n_{i}}$ and summands $C_{i}$ of $G$ satisfying the following:

(a) If $X_{i}=\left\langle x_{n_{i}}\right\rangle$, then $\left|X_{i}\right| \geq p^{i}$;

(b) $C_{i+1} \subseteq C_{i}$;

(c) If $Y_{i}=X_{1} \oplus X_{2} \oplus \cdots \oplus X_{i}$, then $G=Y_{i} \oplus C_{i}$;

(d) $C_{i+1} \cap A_{n_{i}}=C_{i} \cap A_{n_{i}}$.

Before constructing these things, we note that the result easily follows from the following: Claim: If $Y=\oplus_{i} X_{i}$ and $C=\cap_{i} C_{i}$, then $G=Y \oplus C$, so $G$ is not efi.

To verify that (a)-(d) imply the Claim, note first that (b) and (d) imply that $C \cap A_{n_{i}}=$ $C_{i} \cap A_{n_{i}}$. In addition, since $Y_{i} \subseteq A_{n_{i}}$, it follows from (c) that

$$
A_{n_{i}}=Y_{i} \oplus\left(C_{i} \cap A_{n_{i}}\right)=Y_{i} \oplus\left(C \cap A_{n_{i}}\right) .
$$


Taking unions, we can conclude that $G \subseteq Y+C$. Also, if $y=c \in Y \cap C$, then there is an index $i$ such that $y \in Y_{i}$. And since $c \in C \subseteq C_{i}$, (c) implies that $c=y=0$.

So, to finish off the proof, we need to show that once we have constructed $n_{1}, \ldots, n_{i}$; $X_{1}, \ldots, X_{i}$, and $C_{1}, \ldots, C_{i}$ satisfying (a)-(d), we can construct $n_{i+1}, x_{i+1}$ and $C_{i+1}$.

Let $B=C_{i} \cap A_{n_{i}}$, so $A_{n_{i}}=Y_{i} \oplus B$. It follows that $B$ is pure in $A_{n_{i}}$, so that $B$ is also pure in $G$. In addition, $C_{i} / B=C_{i} /\left(C_{i} \cap A_{n_{i}}\right) \cong\left[A_{n_{i}}+C_{i}\right] / A_{n_{i}}=G / A_{n_{i}}$, and since $A_{n_{i}}$ is nice in the reduced group $G$, the factor-group $G / A_{n_{i}}$ is reduced as well - see, for example, [9, Exercise 2(a) from Section 79]. Hence, by assumption, $G / A_{n_{i}}$ is both reduced and unbounded.

Let $E$ be a subgroup of $C_{i}$ containing $B$ such that $E / B$ is a cyclic summand of $C_{i} / B$ of order at least $p^{i}$. It follows that there is a subgroup $C_{i+1}$ of $C_{i}$ containing $B$ such that $C_{i} / B=$ $(E / B) \oplus\left(C_{i+1} / B\right)$. Now, since $B$ is pure in $G$, it is certainly pure in $E$, and since the quotient $E / B$ is cyclic, $E=\left\langle x_{i+1}\right\rangle \oplus B$ for some $x_{i+1} \in E$. Choose $n_{i+1}>n_{i}$ such that $x_{i+1} \in A_{n_{i+1}}$.

Note that (a) and (b) are evident. To verify (d), notice that $C_{i+1} \cap A_{n_{i}}=C_{i+1} \cap C_{i} \cap A_{n_{i}}=$ $C_{i+1} \cap B=B=C_{i} \cap A_{n_{i}}$.

As for (c), note that if $X_{i+1}=\left\langle x_{i+1}\right\rangle$, then $C_{i}=E+C_{i+1}=\left(X_{i+1}+B\right)+C_{i+1}=X_{i+1}+C_{i+1}$. And if $z \in X_{i+1} \cap C_{i+1}$, then $z+B \in(E / B) \cap\left(C_{i+1} / B\right)=\{0\}$, so that $z \in X_{i+1} \cap B=\{0\}$. Therefore, $C_{i}=X_{i+1} \oplus C_{i+1}$, and

$$
G=Y_{i} \oplus C_{i}=Y_{i} \oplus\left(X_{i+1} \oplus C_{i+1}\right)=Y_{i+1} \oplus C_{i+1},
$$

showing that (c) holds, and concluding the proof.

Remark. The condition for niceness on subgroups of the whole group is not necessary in Theorem 2.1. In fact, we do not even need that each $A_{n}$ is nice in $G$; it will suffice to assume that every quotient group $G / A_{n}$ is not the direct sum of a bounded group and a divisible group. However, a little more work must be done.

Theorem 2.1 and its proof are reminiscent of the characterization of groups that are Cdecomposable contained in ([14], Theorem 2.1). Similarly, for purposes of comparison, we state a result that was established as part of the proof of ([8], Theorem 1.3). To begin, if $A$ is pure in $G$, it is easy to check that $G / A$ is bounded iff there is an integer $0<m<\omega$ such that $\left(p^{m} G\right)[p] \subseteq A$.

Theorem 2.2. If $G$ is a separable group, then $G$ is thick iff whenever $G=\cup_{n<\omega} A_{n}$, where $A_{n} \subseteq$ $A_{n+1} \leq G$ and each $A_{n}$ is nice in $G$, there exists an index $k$ such that $\left(p^{m} G\right)[p] \subseteq A_{k}$ for some natural $m<\omega$.

In other words, to characterize thick groups, as opposed to efi groups, we restrict "reduced" in Theorem 2.1 to "separable" and we only demand that the subgroups $A_{n}$ be nice in $G$ (i.e., we drop the assumption of purity). 
We will now raise some questions.

Problem 2.3. If $P$ be a pure subgroup of a group $A$ and both $P$ and $A / P$ are efi, does it follow that $A$ is also efi?

This question can be resolved positively if the following holds: If $A$ is a group with $\Sigma$ cyclic summand of final rank $\alpha, P$ is a pure subgroup of $A$ and $A / P$ does not have such a summand, then $A$ has a summand contained in $P$ which is a $\Sigma$-cyclic group of final rank $\alpha$. For thick groups this question was settled in [15].

In [11], a group $A$ of final rank $\alpha$ was called far from thick if it admits an $\alpha$-homomorphism, i.e., a homomorphism $\phi: A \rightarrow C$, where $C$ is a $\Sigma$-cyclic group, such that for all $n<\omega$ the image $\phi\left(\left(p^{n} A\right)[p]\right)$ has rank at least $\alpha$.

We pause for the following observation:

Proposition 2.4. Let $G$ be a group such that $\left|p^{\beta} G\right|<\operatorname{fin} \mathrm{r}(G)$ for some ordinal $\beta$. Then the group $G$ is far from thick iff the factor-group $G / p^{\beta} G$ is far from thick.

Proof. Using the language of [13], if $\alpha=\operatorname{fin} \operatorname{r}(G)$, then the canonical epimorphism $G \rightarrow G / p^{\beta} G$ is $\alpha$-bijective, i.e., its kernel and cokernel have rank less than $\alpha$. The result therefore follows from ([13], Corollary 2.5).

Now we can ask a question similar to Problem 2.3 for groups that are far from thick.

Problem 2.5. If $P$ be a pure subgroup of a group $A$ and $A$ is far from thick, does it follow that (at least one of) $P$ or A/P is also far from thick?

To establish this we need to prove that if there is an $\alpha$-homomorphism defined on $A$, then the same will hold for either $P$ and $A / P$.

We close this section with the following.

Problem 2.6. Describe the efi groups that are far from thick.

For example, in [3] a $p^{\omega+2}$-projective group was constructed that is efi. Utilizing ([11], Corollary 25), this group will be far from thick.

\section{Groups with efi socles}

Translating the above notions to the category of valuated vector spaces, the socle $G[p]$ of a group $G$ will be said to be essentially finitely indecomposable, or again efi for shortness, if it is not isometric (i.e., isomorphic as a valuated vector space) to a valuated direct sum $V \oplus F$, 
where $F$ is a free valuated vector space with $F(n) \neq 0$ for each $n<\omega$. In other words, $G[p]$ is efi if it fails to possess a valuated summand that is isometric to the socle of an unbounded $\Sigma$-cyclic group.

On the other hand, if $A$ is a group, $A[p]$ is said to be countably-complete if whenever $A[p]=\cup_{n<\omega} A_{n}$, where $A_{n} \subseteq A_{n+1} \leq A[p]$ with each $A_{n}$ being closed in $A[p]$, then there exists an index $k$ such that $\left(p^{m} A\right)[p] \subseteq A_{k}$ for some $m<\omega$.

If $A$ is a separable group, then $A[p]$ is countably-complete iff it is efi (see [7], Proposition 4.4). A different proof of this fact can be based upon Theorem 2.1:

Proposition 3.1. If $A$ is a separable group, then $A[p]$ is countably-complete iff it is efi.

Proof. It is easy to see that if $A[p]$ is not efi, then it is not countably-complete. So assume $A[p]$ is not countably-complete; let $\left\{A_{n}\right\}_{n<\omega}$ testify to this assertion. If $B$ is a basic subgroup of $A$, then we can view $A$ as a pure subgroup of the torsion-complete group $\bar{B}$. Since a torsioncomplete group is quasi-complete (see section 74 of [9] for a definition of this term), then repeatedly using ([9], Theorem 74.1) we can construct an ascending sequence $\left\{P_{n}\right\}_{n<\omega}$ of pure subgroups of $\bar{B}$ such that $P_{n}[p]=A_{n}$ for each $n<\omega$. If $G$ is the union of the $P_{n}$, it is readily checked that each $G / P_{n}$ will be an unbounded separable group. So by Theorem 2.1, $G$ is not efi; i.e., $G$ has a summand $C$ that is an unbounded $\Sigma$-cyclic group. So $C[p]$ will be an unbounded free valuated summand of $A[p]=G[p]$, so that $A[p]$ is not efi, as required.

This can be extended to arbitrary (not necessarily separable) groups via the following argument.

Proposition 3.2. If $A$ is any group, then $A[p]$ is countably-complete iff it is efi.

Proof. Again, clearly if $A[p]$ is not efi then it not countably-complete. So assume $\left\{A_{n}\right\}_{n<\omega}$ demonstrates that $A[p]$ is not countably-complete. If $H$ is a high subgroup of $A$ (i.e., maximal with respect to the property $\left.H \cap p^{\omega} A=\{0\}\right)$, then there is an isometry $A[p] \cong H[p] \oplus\left(p^{\omega} A\right)[p]$. Using $\left\{A_{n} \cap H[p]\right\}_{n<\omega}$, it is easy to check that $H[p]$ also fails to be countably-complete. So with the aid of Proposition 3.1, $H[p]$ has an unbounded free valuated summand. Since this will also be a valuated summand of $A[p]$, it follows that $A[p]$ is not efi, as desired.

We pause now for a quick observation about direct sums.

Proposition 3.3. If $A=B \oplus C$, then $A[p]$ is efi iff both $B[p]$ and $C[p]$ are efi.

Proof. Suppose first that one of $B[p]$ or $C[p]$ fails to be efi. It follows that one of them has a free unbounded valuated summand. Since this will also be a free unbounded valuated summand of $A[p], A[p]$ fails to be efi as well. 
Conversely, assume that both $B[p]$ and $C[p]$ are efi; thus by Proposition 3.2 they are countably-complete. To show $A[p]$ is efi, i.e., countably-complete again in virtue of Proposition 3.2, suppose $A[p]=\cup_{n<\omega} A_{n}$, where $A_{n} \subseteq A_{n+1} \leq A[p]$ and each $A_{n}$ is closed in $A[p]$. Hence $B[p]=\cup_{n<\omega}\left(A_{n} \cap B\right)$ and $C[p]=\cup_{n<\omega}\left(A_{n} \cap C\right)$ with each $A_{n} \cap B$ closed in $B[p]$ and each $A_{n} \cap C$ closed in $C[p]$. Consequently, there are indices $k$ and $l$ and integers $i$ and $j$ such that $\left(p^{i} B\right)[p] \subseteq A_{k}$ and $\left(p^{j} C\right)[p] \subseteq A_{l}$. If $m=\max (i, j)$ and $s=\max (k, l)$, it follows at once that $\left(p^{m} B\right)[p] \subseteq A_{s}$ and $\left(p^{m} C\right)[p] \subseteq A_{s}$. Therefore, $\left(p^{m} A\right)[p]=\left(p^{m} B\right)[p]+\left(p^{m} C\right)[p] \subseteq A_{s}$, as required.

It was proved in [2] that $G$ is efi iff $G / p^{\omega} G$ is efi. So, the following question arises quite naturally: Is $G[p]$ efi iff $\left(G / p^{\omega} G\right)[p]$ is efi? Half of this is straightforward.

Proposition 3.4. If $G[p]$ is efi, then $\left(G / p^{\omega} G\right)[p]$ is efi.

Proof. Suppose $\left(G / p^{\omega} G\right)[p]$ is the ascending union of the closed subgroups $Y_{n} \subseteq\left(G / p^{\omega} G\right)[p]$. If $\phi: G \rightarrow G / p^{\omega} G$ is the canonical epimorphism and we let $X_{n}=\phi^{-1}\left(Y_{n}\right) \cap G[p]$, then $G[p]$ is the ascending union of the subgroups $X_{n}$, which will all be closed in $G[p]$. So there are integers $j, k$ such that $\left(p^{j} G\right)[p] \subseteq X_{k}$; thus $\phi\left(\left(p^{j} G\right)[p]\right) \subseteq \phi\left(X_{k}\right) \subseteq Y_{k}$. Since $\phi\left(\left(p^{j} G\right)[p]\right)$ is dense in $\left(p^{j}\left(G / p^{\omega} G\right)\right)[p]$ and $Y_{k}$ is closed, we can conclude that $\left(p^{j}\left(G / p^{\omega} G\right)\right)[p] \subseteq Y_{k}$, as needed.

Unfortunately, the converse of Proposition 3.4 fails. For example, if $B$ is an unbounded $\Sigma$ cyclic group with torsion completion $\bar{B}$ and we let $G=\bar{B} / B[p]$, then it can simply be checked that $\left(G / p^{\omega} G\right)[p] \cong(p \bar{B})[p]$ will be efi, but $G[p]$ will not be.

In [12] it was observed that each separable group with efi socle is thick. We present a different proof of this fact.

Proposition 3.5. If $A[p]$ is countably-complete, then $A$ is thick.

Proof. Let $A / K$ be a $\Sigma$-cyclic group. Express $A / K$ as an ascending union $\cup_{n<\omega}\left(A_{n} / K\right)$, where all $A_{n} / K$ are $p^{n}$-bounded summands of $A / K$. Therefore,

$$
\left[\left(p^{n} A \cap A_{n}\right)+K\right] / K \subseteq p^{n}(A / K) \cap\left(A_{n} / K\right)=\{0\},
$$

so that $p^{n} A \cap A_{n} \subseteq K$. Now, $A / A_{n} \cong(A / K) /\left(A_{n} / K\right)$ is separable, so it plainly follows that $A[p]=$ $\cup_{n<\omega} A_{n}[p]$ where each $A_{n}[p]$ is closed in $A[p]$. Since $A[p]$ is countably-complete, there is an integer $m<\omega$ such that $\left(p^{m} A\right)[p] \subseteq A_{k}$ for some index $k$. So if $j=\max \{k, m\}$, then

$$
\left(p^{j} A\right)[p] \subseteq\left(p^{k} A\right)[p] \cap\left(p^{m} A\right)[p] \subseteq p^{k} A \cap A_{k} \subseteq K .
$$

According to [1], this shows that $A$ is thick. 
Corollary 3.6. Groups with efi socles are thick.

Proof. This follows directly from Propositions 3.2 and 3.5.

We now observe that all three notions alluded to above do coincide for pure-complete groups.

Proposition 3.7. If $A$ is a pure-complete group, then $A$ is efi iff $A[p]$ is efi.

Proof. We may certainly assume $A$ is reduced and hence separable. If $A[p]$ is efi, it trivially follows that $A$ is efi (this direction does not use that $A$ is pure-complete). Conversely, suppose that $A[p]=A_{1} \oplus A_{2}$ is a valuated direct sum where $A_{2}$ is unbounded and free. It follows that there exist pure subgroups $P_{1}$ and $P_{2}$ of $A$ such that $A_{1}=P_{1}[p], A_{2}=P_{2}[p]$ and $P_{2}$ is an unbounded $\Sigma$-cyclic group. If $x=y+z \in A[p]$, where $y \in A_{1}[p], z \in A_{2}[p]$, then it is plainly verified that

$$
\mathrm{ht}_{A}(x)=\min \left\{v_{A_{1}}(y), v_{A_{2}}(z)\right\}=\min \left\{\mathrm{ht}_{P_{1}}(y), \mathrm{ht}_{P_{2}}(z)\right\}=\mathrm{ht}_{P_{1} \oplus P_{2}}(x) .
$$

Owing to ([9], 26(h)), the internal direct sum $P_{1} \oplus P_{2}$ is pure in $A$. And since $A[p]=\left(P_{1} \oplus P_{2}\right)[p]$, invoking ([9], 26(i)) we have $A=P_{1} \oplus P_{2}$, showing that $A$ is not efi, as expected.

It was established in [1] that a pure-complete group is efi iff it is thick. As an immediate consequence, we obtain the following statement.

Corollary 3.8. If $A$ is a pure-complete group, then $A$ is thick iff $A[p]$ is efi.

Again, in ([12], Proposition 20), a thick group $A$ was constructed such that $A[p]$ is not efi. Hence Corollary 3.8 implies that in such an example $A$ cannot be pure-complete.

\section{Acknowledgement}

The authors are indebted to the referee for the expert suggestions which improved the presentation of the paper as well as they are grateful to the Managing Editor, Prof. Shieh, for his patience and efforts in processing our work.

\section{References}

[1] K. Benabdallah and R. Wilson, Thick groups and essentially finitely indecomposable groups, Can. J. Math. (3), 30 (1978), 650-654.

[2] D. Cutler and J. Irwin, Essentially finitely indecomposable abelian p-groups, Quaestiones Math., 9 (1986), 135-148.

[3] D. Cutler and C. Missel, The structure ofC-decomposable $p^{\omega+n}$-projective abelian p-groups, Commun. Algebra, 12 (1984), 301-319. 
[4] P. Danchev, Commutative group algebras of thick abelian p-groups, Indian J. Pure Appl. Math. (6), 36 (2005), 319-328.

[5] P. Danchev, Notes on essentially finitely indecomposable nonthick primary abelian groups, Commun. Algebra (4), 36 (2008), 1509-1513.

[6] M. Dugas and J. Irwin, On thickness and decomposability of abelian p-groups, Israel J. Math. (2-3), 79 (1992), $153-159$.

[7] P. Danchev and P. Keef, Nice elongations of primary abelian groups, Publ. Mat. (2), 54 (2010), 317-339.

[8] P. Danchev and P. Keef, Nice bases and thickness in primary abelian groups, Rocky Mountain J. Math. (4), 41 (2011), 1127-1149.

[9] L. Fuchs, Infinite Abelian Groups, volumes I and II, Acad. Press, New York and London, 1970 and 1973.

[10] L. Fuchs, Vector spaces with valuations, J. Algebra, 35 (1975), 23-38.

[11] J. Irwin and P. Keef, Primary abelian groups and direct sums of cyclics, J. Algebra, 159 (1993), 387-399.

[12] P. Keef, Primary abelian groups admitting only small homomorphisms, Commun. Algebra (10), 23 (1995), 3615-3626.

[13] P. Keef, Partially decomposable primary abelian groups and the generalized core class property, in Models, Modules and Abelian Groups, Walter de Gruyter, Berlin and New York, 2008, 289-299.

[14] P. Keef, On subgroups of totally projective primary abelian groups and direct sums of cyclic groups, Contemp. Math., 576 (2012), 205-216.

[15] R. Pierce, Homomorphisms of Primary Abelian Groups, in Topics in Abelian Groups, Scott Foresman and Co. (1963), 215-310.

Department of Mathematics, Whitman College, Walla Walla, WA 99362, USA.

E-mail: keef@whitman.edu

Department of Mathematics, Plovdiv University “P. Hilendarski”, Plovdiv 4000, Bulgaria.

E-mail: pvdanchev@yahoo.com 